\title{
EL AFECTO EN RELATOS DE DOLOR CRÓNICO EN COMENTARIOS DE FACEBOOK DE MUJERES CHILENAS
}

\section{AFFECT IN RECOUNTS OF CHRONIC PAIN IN FACEBOOK COMMENTS BY CHILEAN WOMEN}

\author{
Mariana Pascual \\ Pontificia Universidad Católica de Chile \\ mariana.pascual@uc.cl \\ Natalia Díaz Alegría \\ Pontificia Universidad Católica de Chile \\ nydiaz@uc.cl
}

\begin{abstract}
Resumen:
En el marco de los estudios del discurso, esta investigación aborda desde una perspectiva interpersonal el afecto que mujeres chilenas con dolor crónico construyen en sus relatos de experiencias con la endometriosis. Se analizaron 7230 comentarios de Facebook publicados en un período de cuatro años en tres páginas de grupos de apoyo. Los datos fueron clasificados según el tipo de afecto generado, sobre la base del Sistema de Valoración (Martin, English; Martin y White). Posteriormente, estas valoraciones fueron relacionadas con las entidades evaluadas para identificar patrones de uso. Los resultados indican una predominancia marcada de in/felicidad (tristeza) e in/satisfacción (placer y descontento). La tristeza se vinculó significativamente con el dolor, la enfermedad y los tratamientos quirúrgicos. Siendo la tristeza un afecto tan predominante, esta debe ser considerada en el diseño de acciones centradas en las pacientes.
\end{abstract}

Palabras clave: discurso de salud, relatos, afecto, dolor crónico, endometriosis.

\begin{abstract}
:
From a discourse studies perspective, this research examines from an interpersonal standpoint the affect that Chilean women with chronic pain construct in their narratives describing their experiences with endometriosis. A total of 7230 Facebook comments published over a period of four years on three Chilean webpages on endometriosis were analyzed. The data were classified in terms of affect type, following the System of Appraisal (Martin English; Martin and White). Then, these items were related to the appraised entities so as to identify patterns of use. A clear predominance of values of un/happiness (sadness) and dis/satisfaction (pleasure and discontent) was observed. Sadness was significantly related to pain, to the condition per se, and to surgical treatments. Having observed a strong predominance of sadness, this fact should be considered when designing patient-centered health actions.
\end{abstract}

Keywords: health discourse, narrations, affect, chronic pain, endometriosis.

Recibido: 17 de marzo de 2021

Aceptado: 07 de junio de 2021 
Dossier. Mariana Pascual, Natalia Díaz Alegría. "El afecto en relatos de dolor crónico en comentarios de Facebook de mujeres chilenas"

\section{Introducción}

A partir de los años sesenta del siglo XX se han potenciado distintos quehaceres de la práctica clínica, como la capacidad del médico para comprender a los pacientes, en un intento por mejorar su vínculo. Reflejo de ello es el auge de las Humanidades Médicas (Sánchez González), resultante, entre otros factores, de reclamos constantes relacionados con la falta de comprensión de los médicos hacia los pacientes. Esto fue el resultado de una práctica médica científica, centrada en aspectos técnicos, que ignoraba la dimensión de la comunicación. En la actualidad, las revisiones de literatura acusan que esta situación aún persiste en algunos contextos, generando falencias en la atención centrada en el paciente. Algunos estudios reportan que esta problemática se acentúa más si la paciente es mujer (Ramlakham et al.). Esta situación responde parcialmente a la escasa o nula atención que se le ha dado a la comunicación centrada en el paciente en el ejercicio clínico (McCormack et al.), lo cual se vuelve crítico cuando se revisan los testimonios de mujeres a las que les han diagnosticado enfermedades crónicas, como la endometriosis, luego de haber permanecido un largo tiempo sin un diagnóstico claro de su situación de salud. Estas falencias en la comunicación en prácticas de salud plantean importantes desafíos para los estudios del lenguaje.

\subsection{Discursos de salud centrados en el paciente}

La comunicación centrada en el paciente (PCC por su sigla en inglés) nace en Estados Unidos recién en los albores del siglo XXI. Desde las primeras investigaciones, la PCC se ha caracterizado por focalizar la atención en las preferencias, necesidades y valores individuales del paciente, de modo respetuoso. De esta manera, se tiende a garantizar que los valores del paciente guíen las decisiones clínicas, o bien, a interferir en las acciones que el médico determine para el tratamiento del cuadro clínico (Borghi et al.).

El estudio de McCormack y colaboradores (1088) propone que los dominios característicos en la comunicación colaborativa entre médico y paciente son seis: fomentar una relación de curación, intercambiar información, abordar las emociones del paciente, manejar la incertidumbre del diagnóstico y/o tratamiento, tomar decisiones y permitir el 
autocontrol del paciente. A pesar de que visibilizar este modelo de comunicación centrada en el paciente tiene un impacto significativo en la atención clínica, estudios sobre cómo opera la PCC en pacientes mujeres dan cuenta de un escaso uso de las dimensiones mencionadas (Borghi et al.). Resulta sorprendente, entonces, lo poco que se ha avanzado en el trabajo comunicacional sobre el manejo de la incertidumbre del diagnóstico y las emociones que le genera esta situación a la paciente cuando se observa una patología crónica como es la endometriosis.

\subsection{La representación discursiva del dolor: el caso de la endometriosis}

Cabe plantear entonces dónde queda posicionado el dolor que experimenta una mujer con endometriosis dentro de la práctica clínica basada en la PCC. Resulta incuestionable que el dolor es una experiencia física y afectiva que se construye desde el discurso. La noción de dolor, según la International Association for Study of Pain (IASP), se caracteriza por ser una experiencia emocional y sensorial desagradable, asociada a un daño, real o potencial, o descrito en términos de ese daño (Mersky y Bogduk). Esta experiencia sólo puede ser representada mediante el lenguaje cuando se la intenta comunicar tanto para uno mismo como para los demás.

Ahora bien, cuando se experimenta un dolor crónico, lo que se presenta es una experiencia que ha modificado por un tiempo prolongado la vida cotidiana de los pacientes, la cual se comunica a los demás a través de quejas, lamentos y protesta (Kugelmann). En el caso de la endometriosis, la comunicación de la experiencia del dolor por parte de la paciente tiende a quedar relativizada por la frecuente falta de diagnóstico o tratamiento claro. Las investigaciones que indagan la situación de las mujeres a las que se les diagnosticó esta patología revelan que estas pacientes reportan experiencias negativas en su atención médica (Bach et al.). Este dato se torna preocupante cuando se conoce que el 10\% de la población femenina padece esta condición (Quintero, Vinaccia y Quiceno).

La endometriosis, como lo sostienen varias investigaciones (Bach et al.; Quintero, Vinaccia y Quiceno), es una enfermedad que presenta un deterioro importante en la calidad de vida de las mujeres que la padecen. Esto se debe a que tanto el dolor como el funcionamiento social y psicológico de las pacientes son factores que afectan su cotidianidad. Puntualmente, el dolor que se experimenta lleva a que estas pacientes deban 
Dossier. Mariana Pascual, Natalia Díaz Alegría. "El afecto en relatos de dolor crónico en comentarios de Facebook de mujeres chilenas"

disminuir sus actividades de rutina, tanto laborales como educativas, lo que repercute en su calidad de vida (Lagana et al.; Facchin et al.). Dada esta situación, una atención centrada en la paciente con foco en su comunicación sobre el dolor resulta fundamental en el manejo de la patología.

En este trabajo abordamos el fenómeno de las experiencias con el dolor desde una perspectiva sociolingüística. Esto implica entender al lenguaje en su vinculación con otros campos de investigación (van Dijk), como un sistema de generación de significados que solo puede ser comprendido acabadamente si es considerado en función del contexto social en el que ocurre. Es así que nuestra práctica de investigación se sustenta en el área específica de los estudios del lenguaje desde una perspectiva discursiva (Halliday; Fairclough). Asimismo, enfatizamos el carácter funcional del lenguaje, es decir, aquello que el individuo puede hacer socialmente con él. De esta manera, el análisis se centra en el tipo de significados que el lenguaje genera y el propósito que cumple, más que en las estructuras internas del sistema de la lengua, como sería el caso de las perspectivas formales. La lengua es considerada aquí como un sistema semiogénico, es decir, que tiene el potencial de generación de significados. Es quizás uno de los sistemas semióticos más poderosos y creativos que existen en nuestras sociedades.

\subsection{El afecto como modo de realización de la actitud ante el dolor}

Los individuos generan significados haciendo uso del lenguaje permanentemente, y lo hacen con distintas finalidades. Desde la propuesta teórico-analítica de la Lingüística Sistémico Funcional (Halliday y Matthiessen) se consideran tres funciones principales: para representar el mundo y la experiencia; para construir y consolidar relaciones interpersonales y para estructurar nuestros mensajes de modo tal que constituyan textos de formatos comprensibles y adecuados a los contextos de uso. La segunda funcionalidad es la que concentra la atención de este trabajo, la denominada dimensión interpersonal del lenguaje, por medio de la cual los individuos construyen relaciones con otros individuos, expresan sus emociones y sus posicionamientos con respecto a sus interlocutores y hacia el mensaje, en un proceso de negociación que tiende a alinear a los participantes de una interacción para construir relaciones de armonía (Martin, Beyond). 
En particular, uno de los tipos de significados que cobra relevancia en los relatos de enfermedades como los que aquí analizamos es el que se identifica como afecto, una de las categorías de significados que forman parte junto con el juicio y la apreciación de las dimensiones de la Actitud, elemento fundamental en la construcción de la Valoración (Bullo, Exploring, "I feel"). Tradicionalmente, los elementos que se consideraban evaluativos en el lenguaje eran los adjetivos (ej. doloroso, terrible, inolvidable). Sin embargo, a lo largo de las décadas, se han realizado numerosos aportes que dan cuenta de la enorme variedad de recursos lingüísticos que utilizan los individuos para expresar la evaluación en el discurso. El afecto, como subcategoría de la Actitud, se subdivide a su vez en des/inclinación (lo que se teme y se desea), in/felicidad, in/satisfacción e in/seguridad (Martin, English, Beyond; Martin y White). Resulta pertinente también definir aquí la noción de entidades valoradas (Hood), en tanto constituye una codificación del afecto que hace referencia al elemento que lo generó. Así, en el caso de "mi primera cirugía es en septiembre y tengo miedo de que me dejen peor de lo que estoy" estamos frente a un caso de codificación de afecto de tipo inclinación, en el que la paciente construye su miedo ante un resultado incierto, el que se configura como entidad valorada.

Los resultados de estudios previos (Bullo, Exploring, "I feel"; Pascual, La construcción, Discurso) han dado cuenta de la enorme utilidad de los relatos de las pacientes en investigaciones en las que el foco de las indagaciones es la paciente, a cuya experiencia se accede a través de sus narrativas (Antón y Goering). El valor de las narrativas como forma de construir discursivamente la experiencia en todos sus aspectos ha sido reportada y caracterizada desde múltiples perspectivas. En este sentido, el análisis del discurso se constituye como arena propicia para investigaciones sobre la construcción de la experiencia (Martin y Plum).

\subsection{La construcción del dolor en redes sociales: el caso de Facebook}

Las redes sociales constituyen valiosas fuentes de datos para indagaciones de diversas disciplinas (Richardson). El fenómeno de la construcción de narrativas en modalidades digitales se ha visto propiciado por el hecho de que los individuos tienden a

\footnotetext{
1 Los ejemplos han sido tomados textualmente del corpus. En consecuencia, pueden incluir errores ortográficos o gramaticales, a fin de respetar la manera en la que fueron escritos originalmente.
} 
Dossier. Mariana Pascual, Natalia Díaz Alegría. "El afecto en relatos de dolor crónico en comentarios de Facebook de mujeres chilenas"

compartir sus experiencias íntimas, amparados por una relación "limpia, pura, libre de contaminaciones con la tecnología de la computadora" (Koteyko y Hunt). A pesar de la enorme potencialidad de creación de significados por signos no-lingüísticos, la interacción en grupos como Facebook mantiene la característica principal de desarrollo a través del texto, pero oculta otra enorme lista de elementos denotativos de la identidad, y solo presentes en la interacción cara a cara, como la vestimenta, postura corporal, tono de voz, gesticulaciones, entre muchos otros.

En esta misma línea, se ha argumentado a favor del carácter democratizador que identifica a las interacciones en grupos online, en las cuales no hay una marcada evidencia de voces que representen autoridades, y que lideren la interacción. Asimismo, ofrecen un espacio para que el foco de la interacción sea quienes padecen los problemas que se construyen en el discurso. Estos rasgos distintivos de la interacción y generación de redes online señalan a los comentarios de Facebook como una herramienta de comunicación con grandes beneficios terapéuticos potenciales (Hardey; Hardey y Koteyko). La relevancia y popularidad de estas configuraciones de interacciones postulan grandes desafíos para los estudios de vínculos entre el lenguaje y su impacto en la salud.

En el presente estudio nos propusimos como objetivo determinar cómo mujeres chilenas con dolor crónico construyen discursivamente el afecto en relación con sus experiencias clínicas con la endometriosis en relatos de comentarios de Facebook, sobre la base de dos ejes de indagación principales: (a) el tipo de afecto que codifican y (b) las entidades que gatillan esas reacciones afectivas.

\section{Metodología y análisis}

Este trabajo fue abordado desde una metodología mixta (Bornat; Elliott; Tashakkori y Teddlie), que combinó procesos de acercamiento y recolección de datos auxiliados por herramientas digitales y un análisis de los datos tanto cuantitativo como cualitativo de tipo manual, con la asistencia del software de clasificación Nvivo-New (versión 2020). Este programa ha sido considerado como una de las herramientas más potentes para el análisis de datos cualitativos porque ofrece la posibilidad de trabajar múltiples fuentes de datos, como entrevistas, grupos de discusión, encuestas, medios de comunicación social o páginas web (Robins y Eisen). 
Se recolectaron la totalidad de los comentarios realizados en las tres páginas de Facebook chilenas de acceso abierto, relacionadas con la endometriosis, con un número de seguidores igual o mayor a 750. Se seleccionaron las tres páginas que cumplían con este criterio: Fuchen (Fundación Chilena de Endometrisis) ${ }^{2}$, Endo Chile ${ }^{3}$ y Endometriosis en Chile ${ }^{4}$. Se utilizó el software Ncapture for Nvivo para la captura de los comentarios emitidos durante un período en el que las tres páginas estuvieron activas de manera simultánea, entre enero de 2016 a marzo de 2020. La fecha de corte del período se determinó en función del inicio de la pandemia Covid-19, que marcó un giro abrupto evidente en los contenidos publicados.

Se recolectaron tanto los datos lingüísticos como los no-lingüísticos, que consistieron fundamentalmente de gráficos, fotos, emoticones, gifs y otros recursos semióticos icónicos típicos de redes sociales (Stæhr). Para los propósitos de este trabajo nos limitaremos a los de naturaleza lingüística. La unidad de análisis consistió en comentarios de pacientes de endometriosis cuya función es la de transmitir sus experiencias con la enfermedad. El corpus inicial estaba constituido por un total de 3057 comentarios, formados por 69.944 palabras. Esta cifra corresponde al número depurado de palabras, capturados por Nvivo. Esta captura no incorpora ni emoticones ni cifras.

El análisis de los datos se realizó de forma manual, con la asistencia del software Nvivo-New para la generación de clasificaciones, categorías y redes semánticas. El análisis incluyó la identificación de las siguientes categorías: Tipo de realización del afecto (directa o indirecta) ${ }^{5}$; Entidad valorada (desencadenante del afecto); Polaridad (afecto positivo o negativo) y tipo de afecto construido. Los tipos de afecto se clasificaron siguiendo la propuesta del Sistema de Valoración (Martin Text; Martin y White), en sus dimensiones de afecto, cuyas categorías presenta la tabla 1. Cabe aclarar que la des/inclinación hace referencia a dimensiones de afecto que surge ante algo posible, mientras que el resto de las

\footnotetext{
${ }^{2}$ https://www.facebook.com/search/top?q=fundaci\%C3\%B3n\%20chilena\%20de $\% 20$ endometriosis

${ }^{3} \mathrm{https}: / / \mathrm{www}$. facebook.com/search/top?q=endo $\% 20$ chile

${ }_{5}^{4} \mathrm{https}$ ://www.facebook.com/EndometriosisEnChile

${ }^{5}$ La codificación del afecto, siguiendo la propuesta de Martin y White a la que adherimos en este trabajo, puede ser directa, cuando la evaluación se inscribe explícitamente a través de términos actitudinales (ej. "No me siento tan sola") o expresada de manera indirecta, o invocada, en la cual el significado actitudinal es sugerido, y el lector es invitado a una determinada interpretación (ej. "no podré ser madre" en contextos de intercambios sobre fertilidad invita a interpretar sentimientos de insatisfacción).
} 
Dossier. Mariana Pascual, Natalia Díaz Alegría. "El afecto en relatos de dolor crónico en comentarios de Facebook de mujeres chilenas"

dimensiones constituyen reacciones afectivas ante entidades de dimensiones de lo real para los sujetos que evalúan.

Tabla 1. Afecto: tipos y subtipo (adaptado de Martin y White con ejemplos extraídos de nuestro corpus)

\begin{tabular}{|c|c|c|c|}
\hline \multirow{14}{*}{ Afecto } & Des/inclinación & Deseo & $\begin{array}{l}\text { Me gustaría que alguna institución me } \\
\text { ayudara. }\end{array}$ \\
\hline & & Miedo & $\begin{array}{l}\text { Tengo miedo de que me dejen peor de lo } \\
\text { que estoy. }\end{array}$ \\
\hline & In/felicidad & Alegría & Ando de muy buen ánimo. \\
\hline & & Antipatía & Los médicos se burlan de uno. \\
\hline & & Tristeza & Lamentablemente el tiempo pasó. \\
\hline & & Estima & Un afectuoso saludo desde Penco. \\
\hline & In/satisfacción & Interés & Quiero saber eso. \\
\hline & & Agrado & Gracias a ella llegué a un especialista. \\
\hline & & Aburrimiento & Ya estaba agotada de todo. \\
\hline & & Descontento & $\begin{array}{l}\text { Hace años vengo aceptándolo para no } \\
\text { deprimirme. }\end{array}$ \\
\hline & In/seguridad & Confianza & $\begin{array}{l}\text { Tengo alguna esperanza de no sentir } \\
\text { más dolor. }\end{array}$ \\
\hline & & Tranquilidad & $\begin{array}{l}\text { Me siento más tranquila estando en sus } \\
\text { manos. }\end{array}$ \\
\hline & & Sorpresa & $\begin{array}{l}\text { Estoy de un par de semanas y aún no lo } \\
\text { creo. }\end{array}$ \\
\hline & & Intranquilidad & $\begin{array}{l}\text { Estoy muy desorientada respecto al } \\
\text { tema. }\end{array}$ \\
\hline
\end{tabular}

Estos dominios del afecto fueron identificados y luego vinculados a las entidades que las motivaron a fin de determinar la configuración de esta dimensión de significados, y acceder a la afectividad de las pacientes. 


\section{Resultados y discusión}

Este estudio propuso como objetivo determinar la configuración discursiva de las experiencias de mujeres chilenas con dolor crónico a partir de sus relatos de experiencias con la endometriosis en páginas de Facebook. A tal fin se analizaron la totalidad de 488 relatos en términos de su construcción directa e indirecta de afecto. A continuación, presentamos los resultados de las categorías generales de afecto identificadas, según el modelo propuesto y al que adherimos (Martin English; Martin y White; Hood).

El total de instancias de afecto detectadas fue de 676. El análisis demuestra una marcada predominancia de dos tipos de afecto: in/felicidad (294 instancias) e in/satisfacción (215 instancias), con porcentajes correspondientes al 43,4\% y 31,8\%, respectivamente. Este primer acercamiento a los datos indica que las experiencias construidas en los relatos emergen de sentimientos afectivos más allegados a los logros a los cuales las pacientes aspiran y a los afectos resultantes de su concreción o falta de concreción. Los ejemplos (1) a (8) ilustran con instancias extraídas del corpus los afectos de cada uno de los tipos presentados ${ }^{6}$.

(1) Hola! No saben la felicidad que siento de encontrar a esta asociación. (C128: Afecto-Felicidad)

(2) Lei tus palabras y solte el llanto... es revivir cada una de mis cirugías... donde no pasaban ni 8 meses y nuevamente en pabellón... soportando dolores indescriptibles... batallando dia a dia por hacer una vida normal "tener que oir que no sirves"....y asi, soportar mil cosas ...y la catalogan como enfermedad benigna... cuando al final destruye tu vida de manera fisica $y$ mental...(C284: Afecto-Infelicidad)

(3) Guauuu que hermoso gesto, a mi me operó XXX hace 10 meses y nada malo que decir excelente profesional y persona... Hasta ahora me he sentido muy bien ... (C322: Afecto-Satisfacción)

(4) Mas de 12 años sin diagnostico. Hace un año me diagnosticaron termine con una hiterctomia en junio y a la fecha nuevamente con dolores horribles (C25:Afecto-Insatisfacción)

(5) ... tendría que hacerme la histerectomía y eso me provoca sentimientos encontrados ya que quisiera tener otro hijo en un futuro no muy lejano y con esto, el doctor me dijo que un tanto dificil pero también están mis deseos de vivir muchos años y ver crecer a mí hija... (C240: Afecto-Inclinación)

\footnotetext{
${ }^{6}$ En los ejemplos se incluye el código del comentario según su aparición en el corpus precedido de la letra "C". Se indican con subrayado las codificaciones explícitas del afecto; en negrita se indican los tipos y subtipos de afecto o entidad valorada, según corresponda.
} 
Dossier. Mariana Pascual, Natalia Díaz Alegría. "El afecto en relatos de dolor crónico en comentarios de Facebook de mujeres chilenas"

(6) Yo tengo adenomiosis y mi primera cirugía es en septiembre y tengo miedo de que me dejen peor de lo que estoy. (C279: Afecto-Desinclinación)

(7) ... yo me operé com el doctor Xxx un 7 muy profesional lo recomiendo mucho (C94: Afecto-Seguridad)

(8) hoy fui por primera vez al medico para explicar mis sintomas y sali totalmente confundida el medico me mandó al gastroenterologo, realmente no se.que pensar mis sintomas van mas alla de una gastritis o un colon irritable, necesito saber sus experiencias por que tengo un dolor pelvico constante, dolor de piernas, y cuando llegan los dias sufro demasiado, al punto de desmayarme... le.agradeceré si alguien me.puede orientar. Algunos dicen que soy alaraca. (C374: Afecto-Inseguridad)

En lo que se refiere a las entidades evaluadas, se identificaron un total de 25 entidades, de las cuales la endometriosis concentró la gran mayoría de las instancias valoradas $(59,3 \%)$. En orden decreciente de frecuencia, y con cifras mucho menores, observamos las instituciones de salud (7\%), el personal de salud $(5,4 \%)$, maternidadinfertilidad $(4,5 \%)$ y conocimiento $(3,8 \%)$. Dada la alta incidencia de los valores de endometriosis, la categoría fue desagregada en subcategorías emergentes. La Figura 1 muestra las entidades vinculadas directamente con la endometriosis que fueron evaluadas.

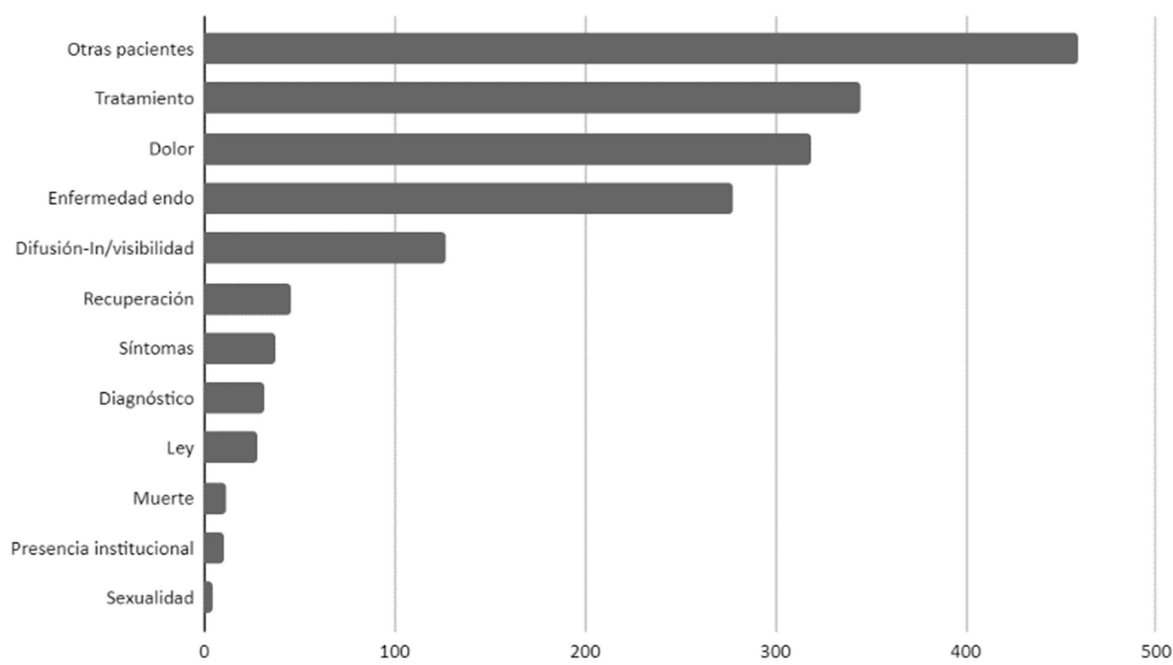

Figura 1. Entidades relativas a endometriosis valoradas en los relatos de las pacientes

Como muestra la figura, los relatos de páginas de Facebook construyen evaluaciones que se vinculan primordialmente a los lazos interpersonales, pero con una 
clara marca valorativa que recae sobre las dimensiones semánticas relativas a tratamiento, dolor y enfermedad per se. La subcategoría difusión también concentró un elevado número de evaluaciones. Los ejemplos (9) a (13) ilustran estas entidades y la manera en la cual las valoraciones se codificaron lingüísticamente.

(9) Es una enfermedad absolutamente invalidante, [...], aún cuando el precio que pagué dejó secuelas en mi vida por siempre, entiendo plenamente a quienes la padecen, pero las mujeres nos caracterizamos por ser valientes, y siempre Dios nos permite salir adelante y criar a nuestros hijos, hoy en día disfruto de dos bisnietos, fuerzas para todas quienes estén sobrellevando está fatídica patología. (C37: Entidad: Otras pacientes)

(10) Yo estoy con tratamiento de acupuntura, llevo 4 sesiones, ademas de yoga, quite de mi alimentacion harina blanca, azucar y leche ya que para la medicina china estos alimentos me perjudican mas por la enfermedad. Me operaron el año pasado y tomaba pastillas anticonceptivas, pero no me tenian muy bien, las deje ... (C65: Entidad: Tratamiento)

(11) Yo tube 3 opeeacion por endometriosis con muchos dolores que pasasa hasta 6 dias hospitalizadas para quitar los dolores ya no habia solucion ni posibilidad de ser madre que decidimos con mi doc xxx (clinica xxx) hacer una histerectomia total ... pero me cambio completamente la vida ya no tengo dolores nada de coctel de pastillas puedo caminar que antes no pidia por los dolores .... (C449: Entidad: Dolor)

(12) Esta enfermedad me ha dañado demasiado fisicamente y emocional tambien ya perdi la esperanza no podre ser madre denuevo es un tema q me duele mucho pero asi es la vida (C5: Entidad: Endometriosis)

(13) Hola, tengo endometriosis diagnosticada a los 30 años, pero vivo con ella desde la adolescencia, fui operada por apendicitis hemorrágica sin saber que era el endometrio que se instaló en el apéndice, segui sin tratamiento hasta que se fue al intestino en donde solamente al operar se dieron cuenta de mi enfermedad que casi me cuesta la vida. ... Es Urgente que está enfermedad se haga visible y que tengamos un apoyo integral. Gracias por hacer visible lo invisible (C9: Entidad: Difusión-In-visibilidad)

Estos resultados indican que las páginas de Facebook se constituyen como espacios para compartir relatos sobre la endometriosis y sus aspectos particulares, como lo son los tratamientos, el dolor y aspectos relativos a la maternidad o infertilidad. Sin embargo, cumplen con la función principal de establecer redes de intercambio afectivo. Esto resulta evidente al observar que la categoría "otras pacientes" fue identificada como la principal fuente de concentración de valoraciones. Fueron utilizadas usualmente para 
Dossier. Mariana Pascual, Natalia Díaz Alegría. "El afecto en relatos de dolor crónico en comentarios de Facebook de mujeres chilenas"

codificar compañía, agradecer su apoyo, expresar deseos de mejora o recuperación, empatía, entre otras demostraciones de afecto.

Seguidamente, los resultados de afecto fueron relacionados con los correspondientes a las entidades valoradas. En función de la marcada predominancia de las categorías in/felicidad e in/satisfacción, los datos fueron desagregados a fin de determinar las subcategorías significativas de cada uno de estos afectos. La Figura 2 presenta los resultados correspondientes a los subtipos de in/felicidad. Como puede observarse, existe una clara tendencia a la construcción de significados vinculados con la tristeza, los cuales ascienden a $75,2 \%$ de la in/felicidad, es decir, un $32,7 \%$ del corpus total. Esta cifra es alarmantemente elevada, lo cual debería alertar a los actores sociales relacionados con esta patología acerca de la necesidad de tomar medidas que apunten de manera directa a contrarrestar esta profunda tristeza.

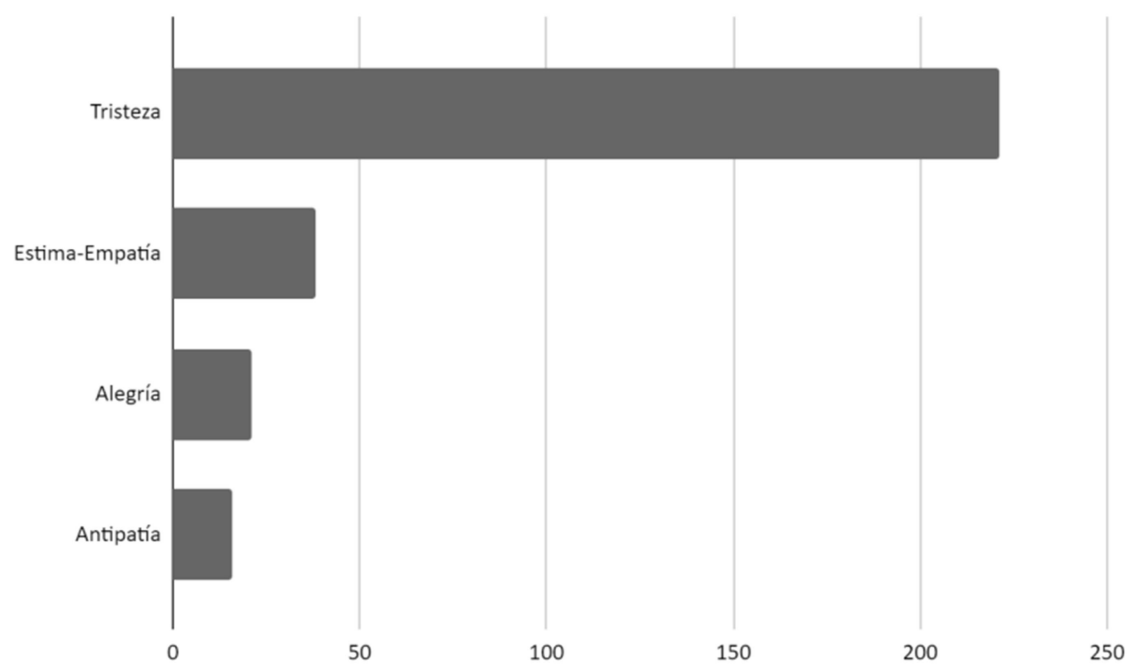

Figura 2. Instancias de in/felicidad identificadas por subtipo

A continuación, se presenta una instancia ilustrativa de este tipo particular de afecto:

(14) ...y y no doy más con ésta enfermedad y estoy recién empezado con todo de nuevo es muy triste $q$ nadie te entienda todos tus dolores y $q$ no tienes ganas de nada muchos te critican q eres una alaraca pero no es asi es una enfermedad real yo no quise tener esto. (C305) 
Los tres subtipos restantes de in/felicidad (estima-empatía, alegría y antipatía) no resultaron de alta frecuencia en el corpus analizado.

El segundo tipo de afecto que prevaleció fue la in/satisfacción, con un total de 215 instancias, un $31,8 \%$ del corpus total. A diferencia de lo observado con los significados de in/felicidad, en esta categoría se visualiza una distribución más homogénea entre dos de los subtipos: placer-agrado y descontento. En relación con el primero, el porcentaje con respecto al corpus total fue de $15,4 \%$, mientas que dentro de la categoría de tipo de in/satisfacción representó el 48,4\%. A su vez, el descontento presentó una frecuencia equivalente al $14,5 \%$ del corpus total y $45,6 \%$ en la dimensión de in/satisfacción, presentadas en la Figura 3.

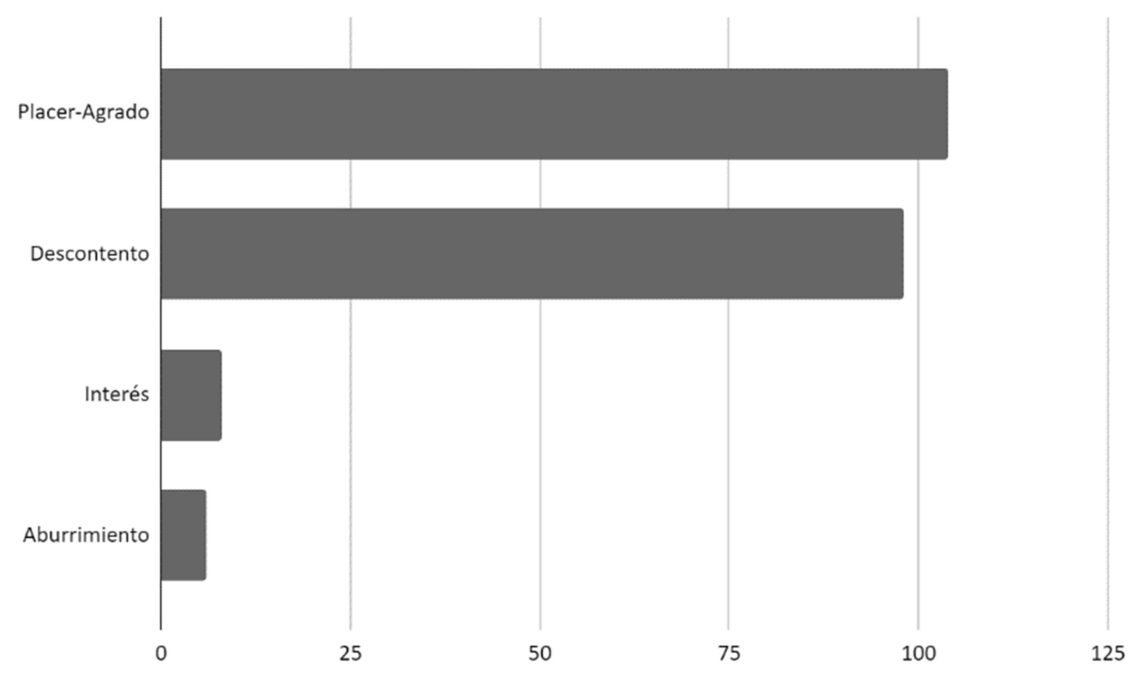

Figura 3. Instancias de in/satisfacción identificadas por subtipo

Estos significados son ejemplificados en las instancias (15) y (16), en las que se destacan los elementos léxico-gramaticales que codifican estos subtipos de afecto:

(15) Yo tuve la suerte de tener unos excelentes médicos de esto hace 30 años. Me operaron 3 veces me dieron drogas y gracias a Dios pese al mal pronostico logre embarazarme y Tenet un embarazo y parto fantástico. (C293 Subtipo de afecto: placer-agrado)

(16) Justamente eso fue lo que ningún doctor me dio me informo y todo paso porque no era un especialista, siempre me decian que si me operaba tenía posibilidad de quedar embarazada, lo cual no era la realidad y una 
Dossier. Mariana Pascual, Natalia Díaz Alegría. "El afecto en relatos de dolor crónico en comentarios de Facebook de mujeres chilenas"

posibilidad para mí. ahora nunca seré madre biológica, nunca podré sentir a una vida que se mueva dentro de mi, solo me queda el consuelo que por algo ocurrió esto ... (C26 Subtipo de afecto: descontento)

Seguidamente, los tipos y subtipos de afecto fueron correlacionados con las entidades a las cuales se vinculaban. Es decir, se establecieron relaciones entre, por ejemplo, la tristeza y los nodos semánticos emergentes que la generaban. Estas relaciones demostraron un nivel de significatividad ${ }^{7}$ en 15 entidades, las cuales fueron reagrupadas en función de su afinidad semántica en un total de seis categorías, que se presentan en la Figura 4.

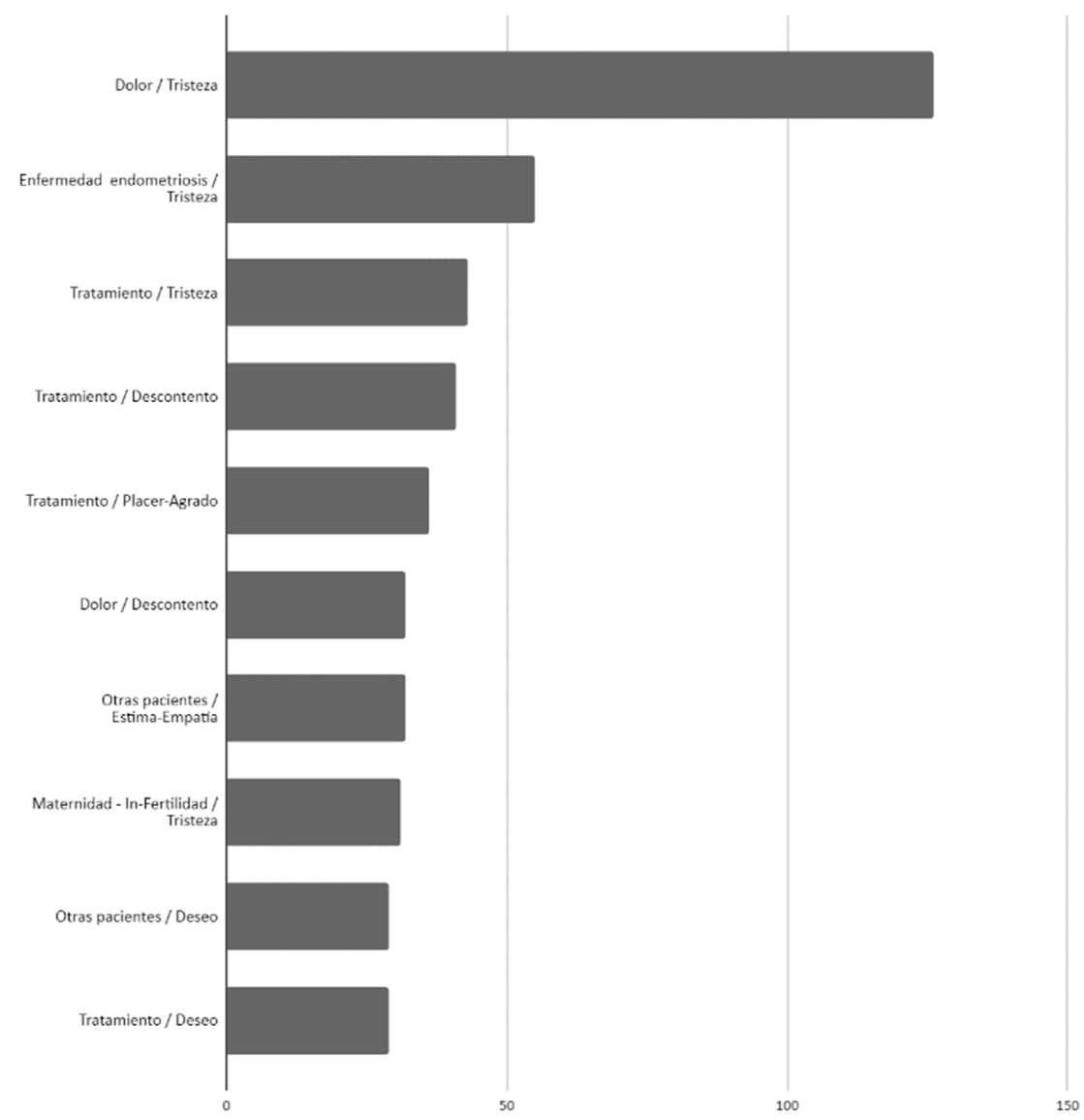

Figura 4. Relaciones entre subtipo de afecto y entidad con la que se vinculan

\footnotetext{
${ }^{7}$ El nivel de significatividad se estableció a partir de las diez combinaciones entre entidades y afectos más frecuentes debido a que desde la sexta y hasta la décima combinación se observó un decrecimiento constante, que cambió a partir de la undécima posición.
} 
También resultaron significativos los vínculos entre otras pacientes y subtipos de afecto de empatía y deseo, confirmando la finalidad social de estos relatos en los que las pacientes se construyen como parte de una comunidad sensible y comprensiva del resto de sus miembros.

Cabe mencionar que algunos relatos no muestran instancias que codifiquen explícitamente alguna valoración, a la vez que otras exhiben múltiples valoraciones y de diferente tipo. Sin embargo, y en consistencia con la literatura (Martin English; Martin y White; Hood), la mera presencia de ciertos elementos ideacionales, que dan cuenta de experiencia, puede resultar suficiente para evocar las valoraciones, como en el siguiente comentario:

(17) A mi me operaron hace 3 meses con histerectomía tenia muchas adherencias sobretodo en el recto lo tenia torcido ahora estoy con tratamiento con unas pastillas para que las células no crezcan y no se ramifique y tratamiento para el dolor. (C315 Dolor evocado)

La mención de la experiencia atravesada evoca una serie de tipos de afecto, no necesariamente explicitados mediante recursos léxico-gramaticales evaluativos, pero que dan cuenta de la variedad de afectos que estas experiencias pueden gatillar, en particular a la luz del contexto sociocultural en el cual se presentan. De hecho, como mujeres latinoamericanas, sabemos de la afectividad negativa que se asocia a atravesar cirugías, a ser histerectomizada, a perder la posibilidad de la maternidad biológica, a no tener certeza sobre la evolución de una enfermedad, entre otras situaciones que culturalmente son asociadas a valores sociales a lograr y consolidar.

Si bien la gravedad del dolor, la infertilidad, las cirugías y diversos tratamientos son lugares comunes con los que pacientes, familiares, amigos y el personal de salud se enfrenta cotidianamente, los resultados de este estudio nos orientan a la toma de acciones en otro sentido. Los relatos de las pacientes indican que el primer padecimiento es la tristeza. En los contextos actuales en los que la paciente cobra una centralidad incuestionable, debemos prestar atención a los discursos que ellas construyen. Es así que, a fin de aportar a esta consigna, hemos reportado el análisis de 488 relatos de pacientes que deben convivir con esta enfermedad. Los datos indican que el tratamiento del dolor, las 
Dossier. Mariana Pascual, Natalia Díaz Alegría. "El afecto en relatos de dolor crónico en comentarios de Facebook de mujeres chilenas"

cirugías y los esfuerzos son de suma gravedad, pero es preciso estar alertas ante el afecto que emerge de sus experiencias y tomar acciones para mejorar su calidad de vida.

\section{Conclusiones}

Los estudios del discurso, planteados desde la intersección de la lingüística y las prácticas sociales, resultan fundamentales para comprender las maneras en que la experiencia se construye textualmente. Esta comprensión resulta de relevancia y urgencia en prácticas sociales fundamentales como lo son las relativas a la salud. Los resultados nos permiten entender la experiencia de aquellos que padecen dolor crónico, y nos dan indicios para encaminar estrategias hacia el mejoramiento de acciones institucionales de salud.

Los resultados de este estudio dan cuenta de una tristeza fuertemente predominante desde las voces de las pacientes. Este es un elemento que ha sido ignorado o al menos subestimado sistemáticamente en las campañas de salud orientadas a pacientes de endometriosis, y a pacientes que sufren de dolor crónico en general. Asimismo, se observan otros focos de insatisfacción, que emergen como resultado del dolor y del padecimiento.

Resulta fundamental estar abiertos a los relatos de las pacientes a fin de diseñar acciones que se orienten a problemáticas reales. Estos resultados pueden arrojar luz para la planificación de acciones tanto en esferas de salud públicas como privadas, con foco en las necesidades de las pacientes. En este sentido, las páginas de Facebook han constituido una fuente particularmente útil de datos que nos han permitido acceder a la profundidad y complejidad de los afectos de las pacientes de endometriosis.

Futuros estudios podrían enfocarse en una profundización de los resultados obtenidos, poniéndolos en perspectiva frente a otras voces de mujeres de otras culturas o expuestas a diferentes presiones socioculturales. También resultaría de interés contrastar los resultados del presente estudio con otras fuentes de información, tales como entrevistas abiertas u otros géneros discursivos que pueden impactar en el tipo y cantidad de afectos reportados, con miras a levantar voces de alerta y provocar cambios en el abordaje de algunas prácticas discursivas en el campo de la salud. 


\section{REFERENCIAS}

Antón, Marta, y Elizabeth M. Goering. Understanding Patients' Voices: A Multi-method Approach to Health Discourse. John Benjamins, 2015.

Bach, Anne Mette et al. "Practices and Attitudes Concerning Endometriosis Among Nurses Specializing in Gynecology". Global Qualitative Nursing Research, vol. 3, 2016, pp. 1-12.

Borghi, Lidia et al. "Patient-centered communication, patient satisfaction, and retention in care in assisted reproductive technology visits". Journal of Assisted Reproduction and Genetics, vol. 36, núm. 6, 2019, pp. 1135-1142.

Bornat, Joanna. "Biographical Methods". Handbook of Social Research Methods, de Pertti Alasuutari et al. SAGE, 2008.

Bullo, Stella. "Exploring disempowerment in women's accounts of endometriosis experiences". Discourse \& Communication, vol. 11, núm. 6, 2018, pp. 1-18.

---. ""I feel like I'm being stabbed by a thousand tiny men": The challenges of communicating endometriosis pain”. Health, vol. 24, núm. 5, 2020, pp. 476-492.

Elliott, Jane. Using Narrative in Social Research: Qualitative and Quantitative Approaches. SAGE, 2005.

Facchin, Federica et al. "Impact of endometriosis on quality of life and mental health: pelvic pain makes the difference". Journal of Psychosomatic Obstetrics \& Gynecology, vol. 36, núm. 4, 2015, pp. 135-41.

Fairclough, Norman. "El ACD como método para la investigación en ciencias sociales". Métodos de análisis crítico del discurso, edición de Ruth Wodak y Michael Meyer. Gedisa, 2003, pp.179-203.

Halliday, Michael A. K. Language as social semiotic: The social interpretation of language and meaning. Edward Arnold, 1978.

Halliday, Michael A. K. y Christian Matthiessen. Construing experience through meaning: A language-based approach to cognition. Cassell, 1999.

Hardey, Michael. "Writing digital selves: Narratives of health and illness on the internet". Representing health: Discourses of health and illness in the media, edición de Martin King y Katherine Watson. Palgrave Macmillan, 2005.

Harvey, Kevin y Nelya Koteyko. Exploring health communication: Language in action. Routledge, 2013.

Hood, Susan. Appraising research: Evaluation in academic writing. Palgrave Macmillan, 2010.

Koteyko, Nelya, y Daniel Hunt. "Performing health identities on social media: An online observation of Facebook profiles". Discourse, Context \& Media, vol. 12, 2016, pp. 59-67.

Kugelmann, Robert. "Complaining about chronic pain”. Social Science \& Medicine, vol. 49, núm.12, 1999, pp. 1663 - 1676.

Laganà, Antonio Simone et al. "Anxiety and depression in patients with endometriosis: impact and management challenges". International Journal of Women's Health, vol. 16, núm. 9, 2017, pp. 323-330.

Martin, James R. English Text: System and structure. Benjamins, 1992. 
Dossier. Mariana Pascual, Natalia Díaz Alegría. "El afecto en relatos de dolor crónico en comentarios de Facebook de mujeres chilenas"

---. "Beyond exchange: Appraisal systems in English". Evaluation in text: Authorial stance and the construction of discourse, edición de S. Hunston y G. Thompson. Oxford University Press, 2000, pp. 142-175.

Martin, James R. y Guenther Plum. "Construing experience: Some story genres". Journal of Narrative and Life History, vol 7, núm.1-4, 1997, pp. 299-308.

Martin, James R. y Peter R. White. The language of evaluation. Appraisal in English. Palgrave Macmillan, 2005.

McCormack, Lauren A. et al. "Measuring patient-centered communication in cancer care: A literature review and the development of a systematic approach". Social Science \& Medicine, vol. 72, núm. 7, 2011, pp. 1085-1095.

Mersky, Harold and Nikolai Bogduk. Classification of chronic pain: descriptions of chronic pain sindromes and definition of chronic pain terms. $2^{\circ}$ ed., International Association for the Study of Pain Press, 1994.

Pascual, Mariana. "La construcción de la evaluación en los discursos de memoria y dolor: aportes desde las intersecciones". Métodos de Análisis del Discurso, Perspectivas argentinas, coordinación de Oscar Iván Londoño Zapata y Giohanny Olave Arias Ediciones de la U Bogotá, 2019, p. 177-194.

---. "Discurso, salud e información desde el relato de pacientes de endometriosis". Discurso \& Sociedad, vol. 14, núm. 2, 2020, pp. 422-442.

Quintero, María Fernanda, Stefano Vinaccia, y Japsy Margarita Quiceno. "Endometriosis: Psychological aspects”. Revi Chil Obstet Ginecol, vol. 82, núm. 4, 2017, pp. 447452.

Ramlakhan, Jessica U. et al. "What constitutes patient-centred care for women: A theoretical rapid review". Int J Equity Health, vol. 18, núm. 1, 2019, pp. 1-12.

Richardson, Kay. Internet discourse and health debates. Palgrave Macmillan, 2005.

Robins, Cynthia y Karla Eisen. "Strategies for the Effective Use of NVivo in a Large-Scale Study: Qualitative Analysis and the Repeal of Don't Ask, Don't Tell”. Qualitative Inquiry, vol. 23, núm. 10, 2017, pp. 768-778.

Sánchez González, Miguel Ángel. Humanism and the teaching of medical humanities. Educación Médica, vol.18, núm. 3, 2017, pp. 212-218.

Stæhr, Andreas. "Reflexivity in Facebook interaction - Enregisterment across written and spoken language practices". Discourse, Context and Media, vol. 8, 2015, pp. 3045.

Tashakkori, A. y Teddlie, C., coordinadores. Handbook of Mixed Methods in Social and Behavioral Research. SAGE, 2010.

Van Dijk, Teun. El discurso como interacción social. Gedisa, 2000. 